\title{
Validade da autorreferência de colesterol elevado na cidade de São Paulo, Brasil, e fatores associados à sensibilidade dessa informação
}

\section{Validity of self-reported high cholesterol in the city of São Paulo, Brazil, and factors associated with this information's sensitivity}

\section{Validez del autoinforme de colesterol elevado en la ciudad de São Paulo, Brasil, y factores asociados con la sensibilidad de esta información}

\author{
Mariane de Mello Fontanelli 1 \\ Luana Romão Nogueira 1 \\ Marcela Riccioppo Garcez 1 \\ Cristiane Hermes Sales 1 \\ José Eduardo Corrente 2 \\ Chester Luiz Galvão César 1 \\ Moisés Goldbaum ${ }^{3}$ \\ Regina Mara Fisberg 1
}

doi: $10.1590 / 0102-311 \times 00034718$

\section{Resumo}

O objetivo do presente estudo foi validar a autorreferência de colesterol elevado em residentes de São Paulo, Brasil, e verificar os fatores associados à sensibilidade dessa informação. Foram utilizados dados do Inquérito de Saúde de São Paulo de 2015, estudo transversal de base populacional com amostra probabilística de residentes do município. Foram incluídos 886 indivíduos com informações do questionário estruturado, dosagem de colesterol total e frações ou que referiram tratamento farmacológico do colesterol elevado. A validade da informação autorreferida de colesterol elevado foi determinada por meio da sensibilidade, especificidade, valores preditivos positivo (VPP) $e$ negativo (VPN) e kappa, considerando as dosagens de colesterol total e LDL-c e o tratamento farmacológico como padrão-ouro. Modelos de regressão logística foram realizados a fim de investigar os fatores associados à sensibilidade dessa informação. A sensibilidade da informação de colesterol elevado tendo em conta o colesterol total como referência foi de 50,6\%, a especificidade de 90,19\%, VPP de 51,64\%, VPN de 89,82\% e kappa de 0,41. Considerando o LDL-c como padrão-ouro, a sensibilidade foi 53,52\%, a especificidade $89,93 \%$, o VPP 49,22\%, o VPN 91,39\% e kappa 0,43. Utilizando a dosagem de colesterol total como referência, a idade (OR = 1,69; IC95\%: 1,24-2,29) e o plano de saúde $(O R=2,91$; IC95\%: 1,06-7,99) se associaram à sensibilidade dessa informação. Considerando a dosagem de LDL-c como padrão-ouro, a idade $(O R=1,71$; IC95\%: 1,13-2,57), o tabagismo (OR = 3,33; IC95\%: 1,08-10,27) e o plano de saúde $(O R=3,64$; IC95\%: 1,10-12,08) foram associados à sensibilidade dessa informação. Os resultados indicam baixa sensibilidade e VPP da autorreferência do colesterol elevado em residentes de São Paulo.

Colesterol; LDL-Colesterol; Hipercolesterolemia; Estudos de Validação

\author{
Correspondência \\ R. M. Fisberg \\ Departamento de Nutrição, Faculdade de Saúde Pública, \\ Universidade de São Paulo. \\ Av. Dr. Arnaldo 715, São Paulo, SP 01246-904, Brasil. \\ rfisberg@usp.br \\ 1 Faculdade de Saúde Pública, Universidade de São Paulo, São \\ Paulo, Brasil. \\ 2 Instituto de Biociências, Universidade Estadual Paulista, \\ Botucatu, Brasil. \\ 3 Faculdade de Medicina, Universidade de São Paulo, São Paulo, \\ Brasil.
}




\section{Introdução}

A doença cardiovascular aterosclerótica continua entre as principais causas de morte mundial, ocupando o topo dos contribuintes para os anos de vida perdidos ou vividos com incapacidade (disability-adjusted life-years - DALYs) no ano de 2015 1. Na maioria dos casos, a doença cardiovascular aterosclerótica é produto de diversos fatores de risco causais, e a combinação desses fatores pode resultar em aumento expressivo do risco 2. Considerado um fator de risco clássico para as doenças cardiovasculares, o colesterol elevado acentua a agressão endotelial e contribui para formação da placa aterosclerótica 3 .

Dados obtidos em 61 estudos prospectivos indicam que a redução de 38,6mg/dL de colesterol total foi associada à diminuição de cerca de metade da mortalidade por doença cardiovascular isquêmica em indivíduos entre 40-49 anos, um terço entre 50-69 anos e um sexto entre 70-89 anos 4. Entretanto, ressalta-se que o impacto na redução do risco de mortalidade é dependente não apenas da quantidade de colesterol sérico reduzida, mas também do ponto a partir do qual se deu a redução. Maior impacto é encontrado quando essa parte de concentrações mais elevadas do que quando parte de concentrações mais próximas às faixas de referência 5 . Tais dados reforçam a importância da prevenção e controle desse fator de risco 1.

De acordo com a Organização Mundial da Saúde (OMS), em 2008, a prevalência global de colesterol elevado era 39\%, e a Europa possuía a maior prevalência (54\%), seguida das Américas (48\%) 6 . No Brasil, de acordo com dados autorrelatados, foi registrado que 12,5\% dos indivíduos avaliados na Pesquisa Nacional de Saúde (PNS) de 2013 tinham colesterol elevado ${ }^{7}$. Já os dados do estudo Vigilância de Fatores de Risco e Proteção para Doenças Crônicas por Inquérito Telefônico (VIGITEL) apontam que 22,6\% dos indivíduos relatam ter colesterol total ou triacilgliceróis elevados no conjunto das 27 cidades estudadas, sendo essa prevalência estimada em $21,8 \%$ para a cidade de São Paulo 8 .

Ao considerar a lipoproteína de baixa densidade (low density lipoprotein cholesterol - LDL-c), demonstrou-se que 45,5\% dos indivíduos avaliados na coorte do Estudo Longitudinal de Saúde do Adulto (ELSA-Brasil) apresentavam LDL-c elevado ou faziam uso de medicamentos hipolipemiantes, e, por outro lado, que apenas 58,1\% desses indivíduos conheciam seu diagnóstico 9 .

Na América Latina, estudo de revisão de pesquisas populacionais que avaliou a tendência histórica dos níveis de colesterol observou valores relativamente baixos de diagnóstico, conhecimento, tratamento e controle do colesterol elevado 10. O estudo de Avaliação Múltipla de Fatores de Risco Cardiovascular na América Latina (Cardiovascular Risk Factor Multiple Evaluation in Latin America - CARMELA), por exemplo, demonstrou que somente $41 \%$ dos indivíduos avaliados nas cidades latinas conheciam o diagnóstico de hipercolesterolemia 11. Esse resultado serve de alerta pelo impacto dos níveis de colesterol total e LDL-c nas doenças cardiovasculares, e levanta dúvidas com relação à validade dessa informação autorrelatada, embora a dosagem possa ser inviável em estudos populacionais.

Investigações têm demonstrado que características demográficas, socioeconômicas e de estilo de vida estão relacionadas à sensibilidade da autorreferência de colesterol elevado. Estudo que avaliou a validade dessa informação em 8.236 adultos com dados provenientes da Pesquisa Nacional de Saúde dos Estados Unidos (National Health and Nutrition Examination Survey - NHANES), utilizando como padrão-ouro a dosagem de colesterol total, verificou que indivíduos mais velhos, com excesso de peso, brancos e com a última consulta médica em um período menor de dois anos referiram melhor a condição de hipercolesterolemia 12. Dados de estudo nacional indicaram que indivíduos do sexo feminino, mais velhos e beneficiários de planos de saúde demonstraram melhor conhecimento da condição dessa alteração ${ }^{9}$.

Nesse cenário, ressalta-se a importância de conhecer a validade da informação autorreferida do colesterol elevado, tendo em vista sua relevância como ferramenta para o monitoramento e vigilância do colesterol total e do LDL-c elevados na população, e a inexistência de estudos brasileiros de base populacional que forneçam a informação. O objetivo do presente estudo foi validar a informação de colesterol elevado em adolescentes, adultos e idosos residentes em São Paulo e verificar os fatores associados à sensibilidade dessa informação. 


\section{Material e métodos}

\section{Desenho do estudo e amostragem}

Para o presente estudo, foram utilizados dados do Inquérito de Saúde de São Paulo (ISA-Capital 2015), estudo transversal de base populacional com amostra probabilística de adolescentes, adultos e idosos residentes em domicílios permanentes na área urbana do município. Trata-se da terceira edição do ISA-Capital, cujo objetivo principal é produzir conhecimento sobre diversos aspectos do estado de saúde, estilo de vida e uso de serviços de saúde da população da cidade de São Paulo.

O processo de amostragem do ISA-Capital 2015 foi realizado em dois estágios: setor censitário e domicílio. A amostra foi calculada tendo em vista os domínios geográficos (coordenadorias de saúde) e demográficos (adolescentes de ambos os sexos - 12-19 anos; adultos do sexo masculino - 20-59 anos; adultos do sexo feminino - 20-59 anos; e idosos de ambos os sexos -60 anos ou mais). Todos os indivíduos nos domicílios que pertenciam ao domínio demográfico selecionado foram convidados a participar do estudo. Mais informações foram descritas por Fisberg et al. 13 e Alves et al. 14.

Brevemente, no ISA-Capital 2015, foram realizadas 4.059 entrevistas domiciliares. Desses indivíduos, 901 realizaram avaliação antropométrica e coleta de sangue. Para o presente estudo, foram incluídos 886 indivíduos (288 adolescentes, 297 adultos e 301 idosos) com dosagem de colesterol total ou que referiram tratamento farmacológico do colesterol elevado.

O estudo foi aprovado pelo Comitê de Ética em Pesquisa da Secretaria Municipal de Saúde de São Paulo e da Faculdade de Saúde Pública da Universidade de São Paulo (número 003.0.162.000-08 e CAAE 36607614.5.0000.5421).

\section{Variáveis demográficas, socioeconômicas e do estilo de vida}

Dados demográficos, socioeconômicos, de morbidade referida (hipertensão arterial, diabetes mellitus, colesterol elevado, entre outras), sobre o uso de medicamentos e de estilo de vida (hábito alimentar, atividade física, sono, tabagismo e etilismo) foram coletados por meio de questionário estruturado aplicado por 28 entrevistadores treinados durante a primeira visita domiciliar.

O sexo foi analisado como variável dicotômica (feminino e masculino). A idade foi medida em anos, sendo utilizada de forma contínua e categórica. Para classificação da faixa etária, foram consideradas as definições da OMS e do Estatuto da Criança e do Adolescente.

A raça autorreferida foi classificada em "brancos" e "outras raças" (negros, pardos, amarelos e indígenas). A situação conjugal foi classificada em "com companheiro" (casado e união conjugal estável) e "sem companheiro" (solteiro, separado, divorciado e viúvo).

A escolaridade do chefe de família foi medida em anos de estudo e classificada em "menos de 10 anos de estudo" (Ensino Médio incompleto) e "10 anos de estudo ou mais" (Ensino Médio completo). A renda familiar per capita, calculada por meio da soma da renda de todos os membros da família e dividida pelos mesmos, foi utilizada de forma categórica, "até um salário mínimo" (R\$ 788 no ano de 2015) e "mais de um salário mínimo".

O tabagismo foi coletado mediante questões sobre fumo atual ou pregresso, número de cigarros fumados por dia e exposição à fumaça do cigarro. A classificação em "nunca fumou", "ex-fumante" e "fumante" foi baseada nas seguintes perguntas do questionário estruturado: "O(a) $\mathrm{Sr}$.(a) fuma atualmente ou já fumou (pelo menos 100 cigarros ou 5 maços)?" e "O(a) Sr.(a) fuma atualmente?”.

O consumo de bebidas alcoólicas foi investigado por meio de perguntas sobre bebidas de preferência e experimentação de bebidas alcoólicas sucedida de dez perguntas que compõem o instrumento denominado Alcohol Use Disorder Identification Test. Foram categorizados como "não consumidor" os indivíduos que referiam nunca ter experimentado bebida alcoólica ou não beber atualmente. Para a categoria de "consumidor" foram incluídos indivíduos que referiram ingerir bebida alcoólica com a frequência maior do que zero.

A informação sobre plano de saúde foi obtida por meio da pergunta "O(a) Sr.(a) tem ou já teve convênio ou plano de saúde?". O participante foi classificado como "beneficiário de plano de saúde", caso tenha respondido as opções "tem" ou "já teve". 
Dados sobre a prática de atividade física foram coletados por meio da versão longa do Questionário Internacional de Atividade Física (International Physical Activity Questionnaire - IPAQ) 15. Indivíduos foram classificados em "cumpre" ou "não cumpre a recomendação" segundo diretriz vigente na época do estudo 16 .

Durante a segunda visita domiciliar, foram coletadas medidas antropométricas e de pressão arterial, além da coleta de sangue. Esses procedimentos foram realizados por quatro enfermeiros previamente treinados.

Peso, estatura e circunferência da cintura foram avaliadas em triplicata, de acordo com procedimentos de aferição recomendados pela OMS 17. Os valores médios de peso e estatura foram utilizados para cálculo do índice de massa corporal (IMC), classificado segundo pontos de corte propostos para adolescentes 18, adultos 19, e idosos 20. Para classificação do estado nutricional dos adolescentes utilizou-se o software WHO AnthroPlus (https://www.who.int/growthref/tools/en/).

A pressão arterial foi avaliada durante a segunda visita domiciliar por meio de um monitor de pressão automático calibrado (Omron, modelo HEM-712C; Omron Healthcare, Quioto, Japão). A medida foi realizada nos braços, iniciando-se pelo braço direito, ambos livres de roupas, com o indivíduo sentado e em silêncio, respeitando-se o intervalo de um minuto entre as medições. Uma medida adicional foi obtida no braço com o maior valor de pressão arterial. Se houvesse diferença maior que $10 \%$ entre as medidas, uma terceira medida foi realizada, totalizando quatro medidas 21 . O participante era classificado com hipertensão arterial se a aferição fosse $\geq 140 \mathrm{mmHg}$ de pressão arterial sistólica ou $\geq 90 \mathrm{mmHg}$ para pressão arterial diastólica, quando com 18 anos ou mais. Foi classificado como hipertenso o adolescente cujas pressões arteriais sistólica ou diastólica foram maiores que o percentil 95, considerando idade, sexo e estatura 21.

A glicemia de jejum foi dosada por meio do ensaio enzimático colorimétrico da glicose oxidase reação de Trinder (Cobas; Roche Diagnostics GmbH, Mannheim, Alemanha). Indivíduos com glice$\mathrm{mia} \geq 126 \mathrm{mg} / \mathrm{dL}$ ou em uso de medicamentos foram classificados como com diabetes 22 .

\section{Informação autorreferida de colesterol elevado}

A informação de colesterol elevado foi coletada por meio da pergunta "Algum médico já lhe informou que o(a) Sr.(a) tem colesterol elevado?”, realizada durante a aplicação do questionário estruturado. Os entrevistados que responderam à opção "sim" foram considerados indivíduos que autorreferiram a condição de colesterol elevado.

Para os indivíduos que autorreferiram ter colesterol elevado, também foi questionado a idade em que o primeiro diagnóstico foi realizado, a limitação que essa condição acarreta na rotina do indivíduo e as medidas tomadas por ele para controle dessa alteração: dieta alimentar, atividade física, uso de medicamento, acompanhamento regular.

\section{Diagnóstico de colesterol total e LDL-c elevados}

A coleta de sangue venoso e a investigação sobre o uso de medicamentos foram realizadas durante a segunda visita domiciliar. Os participantes foram orientados via telefone a realizar jejum de pelo menos 12 horas e não superior a 14 horas para alimentos e bebidas (exceto água, e para bebidas alcoólicas, solicitou-se jejum mínimo de 76 horas), e a não praticar atividade física intensa ou realizar esforços físicos exaustivos nas 24 horas precedentes à realização do exame.

A fim de verificar se os participantes aderiram às recomendações para a realização do exame foi aplicado um checklist anterior à coleta de sangue, com questões sobre o horário da última refeição, ingestão de alimentos ou bebidas após a última refeição, ingestão de bebidas alcoólicas e sobre a prática de atividade física no dia anterior. A não adesão implicava o agendamento de nova data para coleta de sangue.

O diagnóstico laboratorial de colesterol total elevado foi determinado por meio da reação de Trinder (colesterol oxidase), e LDL-c foi avaliado por meio do método colorimétrico enzimático homogêneo, ambos utilizando-se reagentes da Cobas (Roche Diagnostics GmbH, Mannheim, Alemanha).

O uso de medicamentos nos últimos dois dias foi investigado a fim de identificar os medicamentos de uso crônico e agudo que pudessem interferir nas dosagens bioquímicas, além de facilitar o relato do 
entrevistado. Em caso afirmativo, o indivíduo era encorajado a mostrar as caixas dos medicamentos para que os entrevistadores registrassem o nome comercial ou o princípio ativo do medicamento, bem como a dosagem em uso. Para medicamentos descritos apenas pelo nome comercial, fez-se a identificação do princípio ativo para posterior classificação do medicamento segundo a indicação.

Para classificação do colesterol total e LDL-c elevados, foram considerados os pontos de corte sugeridos pela diretriz em vigência à época do estudo (adolescentes: colesterol total $\geq 170 \mathrm{mg} / \mathrm{dL}$, LDL-c $\geq 130 \mathrm{mg} / \mathrm{dL}$; adultos e idosos: colesterol total $\geq 240 \mathrm{mg} / \mathrm{dL}$, LDL-c $\geq 160 \mathrm{mg} / \mathrm{dL}$ ) ou o relato do uso de medicamentos hipolipemiantes 23 .

\section{Análises estatísticas}

A população do estudo foi descrita segundo variáveis demográficas e socioeconômicas. As prevalências do colesterol total e do LDL-c elevados foram calculadas para a amostra total e segundo faixa etária. Frequências absolutas e relativas foram utilizadas para comparar o colesterol total e o LDL-c diagnosticados à classificação realizada com base na informação autorreferida.

A validade da informação autorreferida de colesterol elevado, utilizando o colesterol total e o LDL-c como padrão-ouro, foi determinada pela sensibilidade, especificidade e valores preditivos positivo (VPP) e negativo (VPN). A estatística kappa foi calculada a fim de verificar a concordância entre o colesterol total e LDL-c diagnosticados e a informação autorreferida corrigida pelo acaso. A validade da informação também foi verificada segundo características demográficas, socioeconômicas e do estilo de vida. Diferenças significativas entre os valores de sensibilidade, especificidade, VPP e VPN foram encontrados pela não sobreposição do intervalo de 95\% de confiança (IC95\%). A fim de investigar os fatores associados à sensibilidade da informação de colesterol elevado, considerando o diagnóstico de colesterol total e LDL-c elevados como padrão-ouro, foram realizados modelos múltiplos de regressão logística. O acerto da condição de colesterol total ou LDL-c elevados (sim ou não) foi incluído como variável dependente e as variáveis: idade, sexo, escolaridade do chefe de família, renda familiar per capita, tabagismo, consumo de álcool, hipertensão, diabetes e plano de saúde, como variáveis independentes. Os modelos foram aceitos após teste de Hosmer-Lemeshow >0,05.

As análises foram realizadas no software Stata versão 13.0 (https://www.stata.com) considerando o desenho amostral do estudo por intermédio do módulo survey, com exceção da estatística kappa. O nível de significância estabelecido foi $<0,05$.

\section{Resultados}

A amostra do presente estudo foi composta por $24,4 \%$ de adolescentes, $50,2 \%$ de adultos e $25,4 \%$ de idosos; incluiu majoritariamente homens (51,6\%), indivíduos que se autodeclararam brancos $(52,6 \%)$, com escolaridade do chefe de família de 10 anos ou mais $(52,5 \%)$, renda familiar per capita menor do que um salário mínimo $(52,1 \%)$ e usuários de plano de saúde $(61,7 \%)$.

A prevalência de colesterol total elevado diagnosticado foi 17,1\% (IC95\%: 14,7-19,7), sendo 15,1\% (IC95\%: 11,5-19,6) entre adolescentes, 12\% (IC95\%: 9,10-15,7) entre adultos e 25,5\% (IC95\%: 20,830,9) em idosos. Dos 177 indivíduos diagnosticados com colesterol total elevado, apenas 87 (50,6\%; IC95\%: 40,8-60,3) conheciam a condição (Tabela 1). Desses, 89\% (IC95\%: 77,4-95,0) referiram algum tipo de tratamento, e a medicação hipolipemiante foi referida por 75,7\% (IC95\%: 62,9-85,1) (Figura 1).

Já a prevalência de LDL-c elevado diagnosticado foi 15,4\% (IC95\%: 13,0-18,2), sendo 4,13\% (IC95\%: 2,2-7,5) entre adolescentes, 13,4\% (IC95\%: 10,3-17,3) entre adultos e 30\% (IC95\%: 25,0-35,4) em idosos. Dos 150 indivíduos diagnosticados com LDL-c elevado, apenas 81 conheciam a condição (53,5\%; IC95\%: 43,4-63,4) (Tabela 1). Desses, 93,4\% (IC95\%: 83,9-97,4) referiram algum tratamento, e 79,61\% (IC95\%: 65,9-88,7) dos indivíduos relataram uso de medicação hipolipemiante (Figura 1).

Entre os indivíduos que autorreferiram ter o colesterol elevado, a idade média do diagnóstico médico foi de 58,0 anos (IC95\%: 55,7-60,4). Com relação ao impacto do colesterol elevado, 17,8\% (IC95\%: 10,3-28,8) desses indivíduos relataram que o colesterol elevado impõe algum grau de limitação em suas rotinas. 
Tabela 1

Autorrelato e diagnóstico de colesterol elevado de adolescentes, adultos e idosos residentes em São Paulo, Brasil, 2015.

\begin{tabular}{|c|c|c|c|c|c|c|c|c|c|}
\hline \multirow[t]{3}{*}{ Colesterol elevado autorreferido } & \multicolumn{9}{|c|}{ Colesterol elevado diagnosticado no soro } \\
\hline & \multicolumn{3}{|c|}{ Sim } & \multicolumn{3}{|c|}{ Não } & \multicolumn{3}{|c|}{ Total } \\
\hline & $\mathbf{n}$ & $\%$ & IC95\% & $\mathbf{n}$ & $\%$ & IC95\% & $\mathbf{n}$ & $\%$ & IC95\% \\
\hline \multicolumn{10}{|l|}{ Colesterol total * } \\
\hline Sim & 87 & 8,68 & $6,71-11,15$ & 71 & 8,13 & $6,18-10,62$ & 158 & 16,80 & $13,86-20,23$ \\
\hline Não & 90 & 8,47 & $6,70-10,65$ & 627 & 74,72 & $71,32-77,85$ & 717 & 83,20 & $79,77-86,14$ \\
\hline Total & 177 & 17,15 & $14,74-19,69$ & 698 & 82,85 & $80,22-85,20$ & 875 & 100,00 & - \\
\hline \multicolumn{10}{|l|}{ LDL-C ** } \\
\hline Sim & 81 & 8,25 & $6,33-10,70$ & 76 & 8,52 & $6,46-11,14$ & 157 & 16,77 & $13,82-20,19$ \\
\hline Não & 69 & 7,17 & $5,50-9,30$ & 648 & 76,06 & $72,38-79,39$ & 717 & 83,23 & $79,81-86,18$ \\
\hline Total & 150 & 15,42 & $13,01-18,18$ & 724 & 84,58 & $81,82-86,99$ & 874 & 100,00 & - \\
\hline
\end{tabular}

IC95\%: intervalo de 95\% de confiança; LDL-c: lipoproteína de baixa densidade.

* Definido como elevado quando o colesterol total sérico foi $\geq 170 \mathrm{mg} / \mathrm{dL}$ para adolescentes e $\geq 240 \mathrm{mg} / \mathrm{dL}$ para adultos e idosos, conforme sugerido por Xavier et al. 22, ou quando foi relatado o uso de medicamentos hipolipemiante;

** Definido como elevado quando a LDL-c sérica foi $\geq 130 \mathrm{mg} / \mathrm{dL}$ para adolescentes e $\geq 160 \mathrm{mg} / \mathrm{dL}$ para adultos e idosos, conforme sugerido por Xavier et al. 22, ou quando foi relatado o uso de medicamentos hipolipemiantes.

Indicadores da validade da informação autorreferida de colesterol total elevado e LDL-c elevado foram calculados para a população total (Tabela 2) e estratificados segundo características demográficas, socioeconômicas e de estilo de vida (Tabela 3). Destaca-se que o valor da sensibilidade dessa informação foi de 50,6\% para o colesterol total e 53,5\% para o LDL-c. Considerando o colesterol total, houve diferença na sensibilidade entre as faixas etárias e segundo hipertensão arterial. A especificidade diferiu segundo a faixa etária, a renda familiar per capita, tabagismo e diabetes. O VPP diferiu segundo estado nutricional e diagnóstico de hipertensão arterial e o VPN, segundo faixa etária. Utilizando-se o diagnóstico de LDL-c como padrão-ouro, ocorreu diferença na sensibilidade segundo diagnóstico de hipertensão arterial, na especificidade segundo faixa etária, tabagismo e diabetes, no VPP segundo estado nutricional e diagnóstico de hipertensão arterial e no VPN segundo faixa etária, estado nutricional e diagnóstico de diabetes.

No modelo de regressão logística múltiplo, a idade e o plano de saúde foram positivamente associados à sensibilidade da informação de colesterol elevado, quando o diagnóstico de colesterol total elevado é utilizado como padrão-ouro (Tabela 4). Tendo o LDL-c elevado como referência, a idade, ser ex-fumante e ser usuário de plano de saúde foram positivamente associados à sensibilidade do autorrelato de colesterol elevado, após ajuste por variáveis de confusão.

\section{Discussão}

Os resultados do presente estudo indicam baixa sensibilidade e VPP para a informação de colesterol elevado autorreferida na população de São Paulo. Dessa forma, o uso dessa informação pode subestimar a prevalência de colesterol elevado na população estudada e reduzir o poder de associação com outras variáveis devido ao erro de classificação; portanto, sua utilização exige cautela. Porém, as características associadas à sensibilidade do autorrelato de colesterol elevado possibilitam a identificação de subgrupos da população em que essa informação pode ser utilizada com mais segurança, como idosos, ex-fumantes e indivíduos que são ou foram usuários de plano de saúde. E, ao mesmo tempo, revelam o tamanho do desconhecimento do diagnóstico de colesterol elevado na população de São Paulo.

Os indicadores da validade da informação de colesterol elevado autorreferida observados no presente estudo são semelhantes aos reportados na literatura. Estudo que utilizou dados provenientes do NHANES a fim de avaliar a validade da hipercolesterolemia autorreferida em 8.236 adultos, utilizan- 


\section{Figura 1}

Distribuição dos indivíduos diagnosticados com colesterol total elevado (1a) e lipoproteína de baixa densidade (LDL-c) elevada (1b) de acordo com o conhecimento do diagnóstico e tratamento segundo faixa etária. São Paulo, Brasil, 2015.

1a) Colesterol total elevado

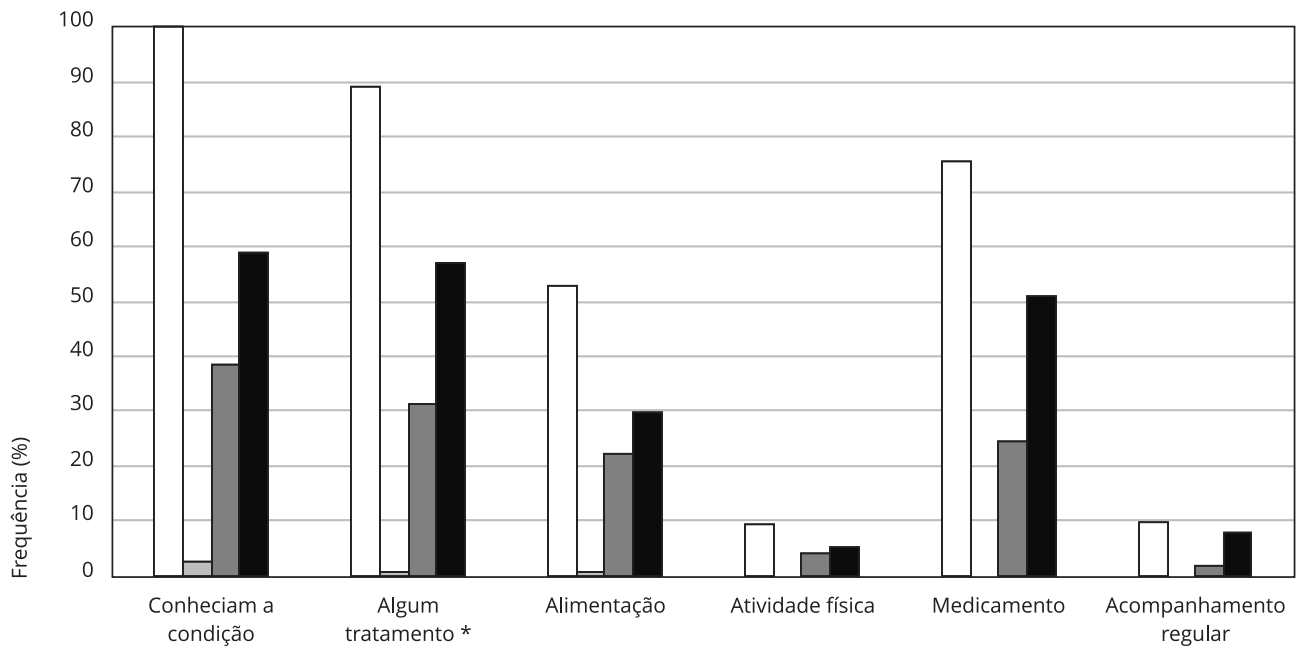

๑ População total

$\square$ Adolescentes

Adultos

Idosos

1b) LDL-c elevado

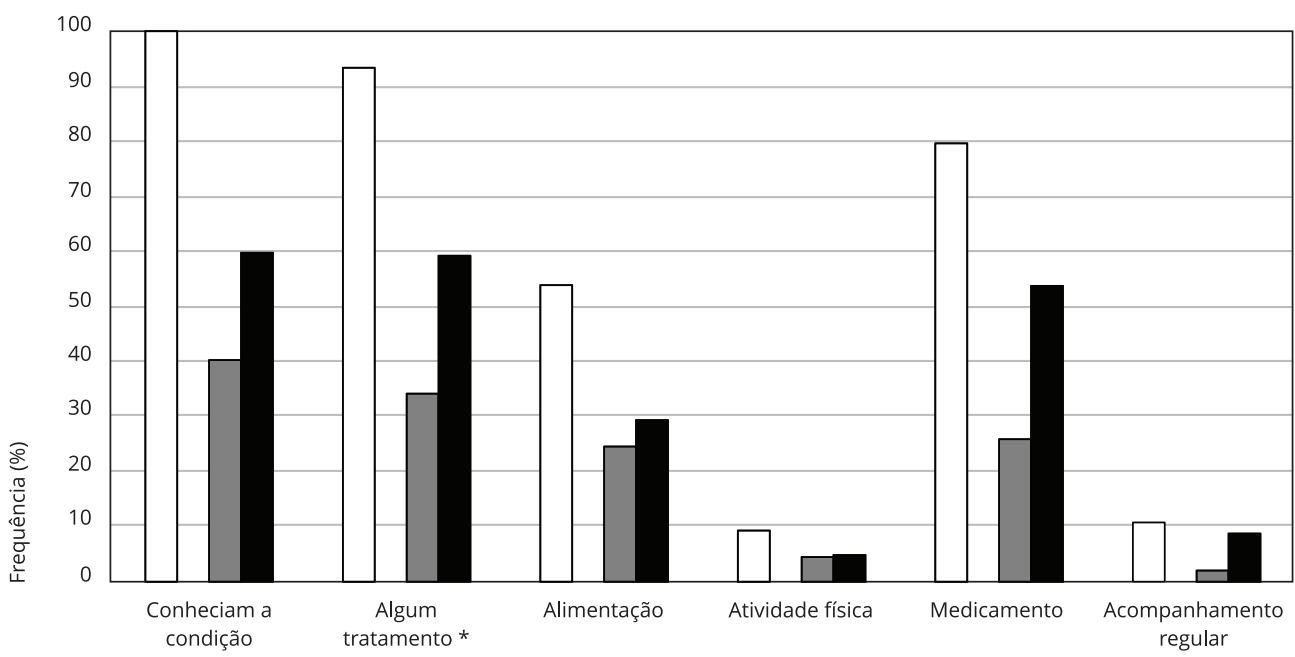

* Algum tratamento: dieta alimentar, atividade física, uso de medicamento ou acompanhamento regular.

do como padrão-ouro a dosagem de colesterol total, estimou sensibilidade em $51 \%$, a especificidade em 89\%, o VPP em 87\% e o VPN em 55\% 12. Em estudo piloto, a Pesquisa Europeia de Saúde (European Health Examination Survey - EHES), realizada em 12 países incluindo 4.127 indivíduos entre 25 e 64 anos, verificou-se para homens e mulheres, respectivamente, sensibilidade de 32 e $30 \%$, especificidade de 95 e 93\%, VPP de 94 e 90\% e VPN de 33 e 40\%, utilizando como padrão-ouro a dosagem sérica de colesterol total 24 . Resultados observados por Newell et al. 25 , que investigaram a validade do autorre- 


\section{Tabela 2}

Sensibilidade, especificidade, valores preditivos positivos (VPP) e negativos (VPN) e coeficiente kappa da informação autorreferida de colesterol elevado de adolescentes, adultos e idosos residentes em São Paulo, Brasil, 2015.

\begin{tabular}{lcccc}
\hline Validade & \multicolumn{2}{c}{ Colesterol total } & \multicolumn{2}{c}{ LDL-c } \\
& $\%$ & IC95\% & $\%$ & IC95\% \\
\hline Sensibilidade & 50,60 & $40,91-60,24$ & 53,52 & $43,38-63,37$ \\
Especificidade & 90,19 & $87,18-92,55$ & 89,93 & $86,80-92,38$ \\
VPP & 51,64 & $42,59-60,58$ & 49,22 & $39,89-58,60$ \\
VPN & 89,82 & $87,29-91,89$ & 91,39 & $88,85-93,39$ \\
Kappa * & 0,41 & $0,33-0,48$ & 0,43 & $0,35-0,51$
\end{tabular}

IC95\%: intervalo de 95\% de confiança; LDL-c: lipoproteína de baixa densidade.

* Não foi considerado o desenho amostral do estudo.

\section{Tabela 3}

Sensibilidade, especificidade, valores preditivos positivo (VPP) e negativo (VPN) da informação autorreferida de colesterol elevado segundo características demográficas, socioeconômicas e do estilo de vida de adolescentes, adultos e idosos residentes em São Paulo, Brasil, 2015.

\begin{tabular}{|c|c|c|c|c|c|c|c|c|}
\hline \multirow[t]{2}{*}{ Características } & \multicolumn{4}{|c|}{ Colesterol total } & \multicolumn{4}{|c|}{ LDL-C } \\
\hline & $\begin{array}{l}\text { Sensibilidade } \\
\text { (IC95\%) }\end{array}$ & $\begin{array}{c}\text { Especificidade } \\
\text { (IC95\%) }\end{array}$ & $\begin{array}{c}\text { VPP } \\
\text { (IC95\%) }\end{array}$ & $\begin{array}{c}\text { VPN } \\
\text { (IC95\%) }\end{array}$ & $\begin{array}{c}\text { Sensibilidade } \\
\text { (IC95\%) }\end{array}$ & $\begin{array}{c}\text { Especificidade } \\
\text { (IC95\%) }\end{array}$ & $\begin{array}{c}\text { VPP } \\
\text { (IC95\%) }\end{array}$ & $\begin{array}{c}\text { VPN } \\
\text { (IC95\%) }\end{array}$ \\
\hline \multicolumn{9}{|l|}{ Sexo } \\
\hline Homens & $\begin{array}{c}44,1 \\
(30,6-58,5)\end{array}$ & $\begin{array}{c}91,3 \\
(87,6-94,0)\end{array}$ & $\begin{array}{c}45,7 \\
(33,4-58,5)\end{array}$ & $\begin{array}{c}90,8 \\
(87,2-93,5)\end{array}$ & $\begin{array}{c}47,1 \\
(33,0-61,8)\end{array}$ & $\begin{array}{c}90,7 \\
(86,7-93,5)\end{array}$ & $\begin{array}{c}39,5 \\
(28,0-52,2)\end{array}$ & $\begin{array}{c}93,0 \\
(89,5-95,4)\end{array}$ \\
\hline Mulheres & $\begin{array}{c}55,5 \\
(44,6-65,8)\end{array}$ & $\begin{array}{c}88,9 \\
(84,5-92,2)\end{array}$ & $\begin{array}{c}56,0 \\
(43,4-67,8)\end{array}$ & $\begin{array}{c}88,7 \\
(85,5-91,3)\end{array}$ & $\begin{array}{c}57,5 \\
(45,1-68,9)\end{array}$ & $\begin{array}{c}89,1 \\
(84,7-92,3)\end{array}$ & $\begin{array}{c}56,3 \\
(43,2-68,5)\end{array}$ & $\begin{array}{c}89,5 \\
(85,9-92,3)\end{array}$ \\
\hline \multicolumn{9}{|l|}{ Faixa etária } \\
\hline Adolescentes & $\begin{array}{c}5,7 \\
(1,4-21,0)\end{array}$ & $\begin{array}{c}98,4 \\
(95,6-99,4)\end{array}$ & $\begin{array}{c}39,6 \\
(9,9-79,8)\end{array}$ & $\begin{array}{c}84,9 \\
(80,4-88,5)\end{array}$ & - & $\begin{array}{c}97,6 \\
(94,6-99,0)\end{array}$ & - & $\begin{array}{c}95,8 \\
(92,3-97,7)\end{array}$ \\
\hline Adultos & $\begin{array}{c}54,6 \\
(37,8-70,4)\end{array}$ & $\begin{array}{c}90,5 \\
(85,6-93,8)\end{array}$ & $\begin{array}{c}44,3 \\
(29,1-60,6)\end{array}$ & $\begin{array}{c}93,5 \\
(90,0-95,9)\end{array}$ & $\begin{array}{c}48,9 \\
(33,5-64,6)\end{array}$ & $\begin{array}{c}90,4 \\
(85,3-93,8)\end{array}$ & $\begin{array}{c}44,1 \\
(29,1-60,3)\end{array}$ & $\begin{array}{c}91,9 \\
(87,9-94,7)\end{array}$ \\
\hline Idosos & $\begin{array}{c}71,4 \\
(59,4-81,0)\end{array}$ & $\begin{array}{c}79,9 \\
(72,3-85,8)\end{array}$ & $\begin{array}{c}58,8 \\
(47,8-69,1)\end{array}$ & $\begin{array}{c}87,4 \\
(81,0-91,9)\end{array}$ & $\begin{array}{c}64,8 \\
(54,2-74,2)\end{array}$ & $\begin{array}{c}78,3 \\
(70,5-84,6)\end{array}$ & $\begin{array}{c}56,8 \\
(46,2-66,8)\end{array}$ & $\begin{array}{c}83,5 \\
(77,3-88,3)\end{array}$ \\
\hline \multicolumn{9}{|c|}{ Raça autorreferida } \\
\hline Branca & $\begin{array}{c}53,6 \\
(41,6-65,1)\end{array}$ & $\begin{array}{c}88,5 \\
(84,0-91,8)\end{array}$ & $\begin{array}{c}50,5 \\
(38,9-62,1)\end{array}$ & $\begin{array}{c}89,7 \\
(85,7-92,7)\end{array}$ & $\begin{array}{c}58,0 \\
(45,2-69,9)\end{array}$ & $\begin{array}{c}89,3 \\
(84,8-92,6)\end{array}$ & $\begin{array}{c}53,6 \\
(41,0-65,7)\end{array}$ & $\begin{array}{c}90,9 \\
(87,0-93,7)\end{array}$ \\
\hline Outras raças & $\begin{array}{c}47,0 \\
(33,3-61,2)\end{array}$ & $\begin{array}{c}92,1 \\
(88,7-94,6)\end{array}$ & $\begin{array}{c}53,9 \\
(40,6-66,6)\end{array}$ & $\begin{array}{c}89,9 \\
(86,2-92,6)\end{array}$ & $\begin{array}{c}46,9 \\
(31,9-62,4)\end{array}$ & $\begin{array}{c}90,7 \\
(86,9-93,4)\end{array}$ & $\begin{array}{c}43,4 \\
(31,4-56,1)\end{array}$ & $\begin{array}{c}91,8 \\
(88,2-94,4)\end{array}$ \\
\hline \multicolumn{9}{|l|}{$\begin{array}{l}\text { Escolaridade do } \\
\text { chefe de família } \\
\text { (anos) }\end{array}$} \\
\hline Menos de 10 & $\begin{array}{c}54,0 \\
(40,0-67,4)\end{array}$ & $\begin{array}{c}90,2 \\
(85,7-93,4)\end{array}$ & $\begin{array}{c}58,8 \\
(46,2-70,3)\end{array}$ & $\begin{array}{c}88,3 \\
(83,8-91,7)\end{array}$ & $\begin{array}{c}51,2 \\
(38,2-64,1)\end{array}$ & $\begin{array}{c}88,5 \\
(83,8-92,0)\end{array}$ & $\begin{array}{c}50,7 \\
(38,2-63,1)\end{array}$ & $\begin{array}{c}88,8 \\
(84,9-91,7)\end{array}$ \\
\hline 10 ou mais & $\begin{array}{c}46,7 \\
(32,2-61,8)\end{array}$ & $\begin{array}{c}89,9 \\
(85,8-93,0)\end{array}$ & $\begin{array}{c}43,6 \\
(30,9-57,2)\end{array}$ & $\begin{array}{c}91,0 \\
(87,5-93,6)\end{array}$ & $\begin{array}{c}57,2 \\
(38,8-73,8)\end{array}$ & $\begin{array}{c}90,8 \\
(86,8-93,8)\end{array}$ & $\begin{array}{c}47,4 \\
(33,4-61,8)\end{array}$ & $\begin{array}{c}93,6 \\
(89,2-96,3)\end{array}$ \\
\hline
\end{tabular}

(continua) 
Tabela 3 (continuação)

\begin{tabular}{|c|c|c|c|c|c|c|c|c|}
\hline \multirow[t]{2}{*}{ Características } & \multicolumn{4}{|c|}{ Colesterol total } & \multicolumn{4}{|c|}{ LDL-c } \\
\hline & $\begin{array}{c}\text { Sensibilidade } \\
\text { (IC95\%) }\end{array}$ & $\begin{array}{l}\text { Especificidade } \\
\text { (IC95\%) }\end{array}$ & $\begin{array}{c}\text { VPP } \\
\text { (IC95\%) }\end{array}$ & $\begin{array}{c}\text { VPN } \\
\text { (IC95\%) }\end{array}$ & $\begin{array}{c}\text { Sensibilidade } \\
\text { (IC95\%) }\end{array}$ & $\begin{array}{l}\text { Especificidade } \\
\text { (IC95\%) }\end{array}$ & $\begin{array}{c}\text { VPP } \\
\text { (IC95\%) }\end{array}$ & $\begin{array}{c}\text { VPN } \\
\text { (IC95\%) }\end{array}$ \\
\hline \multicolumn{9}{|l|}{$\begin{array}{l}\text { Renda familiar } \\
\text { per capita (salário } \\
\text { mínimo) }\end{array}$} \\
\hline Até 1 & $\begin{array}{c}36,9 \\
(24,0-52,0)\end{array}$ & $\begin{array}{c}90,8 \\
(86,2-94,0)\end{array}$ & $\begin{array}{c}42,4 \\
(28,2-58,1)\end{array}$ & $\begin{array}{c}88,7 \\
(84,2-92,1)\end{array}$ & $\begin{array}{c}42,8 \\
(27,7-59,3)\end{array}$ & $\begin{array}{c}90,6 \\
(86,0-93,8)\end{array}$ & $\begin{array}{c}38,5 \\
(24,7-54,4)\end{array}$ & $\begin{array}{c}92,0 \\
(88,3-94,6)\end{array}$ \\
\hline Mais de 1 & $\begin{array}{c}63,3 \\
(50,1-74,8)\end{array}$ & $\begin{array}{c}86,6 \\
(81,2-90,7)\end{array}$ & $\begin{array}{c}55,1 \\
(44,0-65,8)\end{array}$ & $\begin{array}{c}90,1 \\
(85,9-93,1)\end{array}$ & $\begin{array}{c}63,2 \\
(48,1-76,1)\end{array}$ & $\begin{array}{c}86,6 \\
(80,7-91,0)\end{array}$ & $\begin{array}{c}55,1 \\
(42,6-66,9)\end{array}$ & $\begin{array}{c}90,1 \\
(84,4-93,9)\end{array}$ \\
\hline Não respondeu & $\begin{array}{c}36,0 \\
(13,6-66,7)\end{array}$ & $\begin{array}{c}96,7 \\
(90,9-98,9)\end{array}$ & $\begin{array}{c}60,5 \\
(26,9-86,5)\end{array}$ & $\begin{array}{c}91,6 \\
(85,0-95,4)\end{array}$ & $\begin{array}{c}37,3 \\
(14,5-67,8)\end{array}$ & $\begin{array}{c}96,8 \\
(91,0-98,9)\end{array}$ & $\begin{array}{c}60,5 \\
(26,9-86,5)\end{array}$ & $\begin{array}{c}92,1 \\
(86,6-95,4)\end{array}$ \\
\hline \multicolumn{9}{|l|}{ Situação conjugal } \\
\hline $\begin{array}{l}\text { Sem } \\
\text { companheiro }\end{array}$ & $\begin{array}{c}39,8 \\
(28,9-51,8)\end{array}$ & $\begin{array}{c}92,5 \\
(88,6-95,1)\end{array}$ & $\begin{array}{c}52,4 \\
(38,2-66,3)\end{array}$ & $\begin{array}{c}88,1 \\
(85,0-90,7)\end{array}$ & $\begin{array}{c}51,7 \\
(37,3-65,8)\end{array}$ & $\begin{array}{c}92,8 \\
(88,9-95,4)\end{array}$ & $\begin{array}{c}51,3 \\
(36,9-65,6)\end{array}$ & $\begin{array}{c}92,9 \\
(89,7-95,1)\end{array}$ \\
\hline $\begin{array}{l}\text { Com } \\
\text { companheiro }\end{array}$ & $\begin{array}{c}64,2 \\
(50,3-76,1)\end{array}$ & $\begin{array}{c}87,4 \\
(82,3-91,2)\end{array}$ & $\begin{array}{c}51,5 \\
(39,6-63,2)\end{array}$ & $\begin{array}{c}92,2 \\
(88,3-94,8)\end{array}$ & $\begin{array}{c}55,1 \\
(41,7-67,8)\end{array}$ & $\begin{array}{c}86,3 \\
(80,8-90,4)\end{array}$ & $\begin{array}{c}48,0 \\
(36,1-60,2)\end{array}$ & $\begin{array}{c}89,3 \\
(84,6-92,7)\end{array}$ \\
\hline \multicolumn{9}{|l|}{ Tabagismo } \\
\hline Nunca fumou & $\begin{array}{c}45,7 \\
(35,3-56,6)\end{array}$ & $\begin{array}{c}92,1 \\
(88,5-94,6)\end{array}$ & $\begin{array}{c}55,1 \\
(43,0-66,6)\end{array}$ & $\begin{array}{c}88,8 \\
(85,7-91,4)\end{array}$ & $\begin{array}{c}50,7 \\
(39,0-62,2)\end{array}$ & $\begin{array}{c}92,0 \\
(88,4-94,6)\end{array}$ & $\begin{array}{c}53,8 \\
(41,9-65,3)\end{array}$ & $\begin{array}{c}91,0 \\
(87,6-93,6)\end{array}$ \\
\hline Ex-fumante & $\begin{array}{c}61,9 \\
(41,9-78,6)\end{array}$ & $\begin{array}{c}76,2 \\
(65,0-84,7)\end{array}$ & $\begin{array}{c}42,5 \\
(27,8-58,8)\end{array}$ & $\begin{array}{c}87,6 \\
(77,7-93,4)\end{array}$ & $\begin{array}{c}64,9 \\
(44,5-81,1)\end{array}$ & $\begin{array}{c}76,5 \\
(65,4-84,9)\end{array}$ & $\begin{array}{c}41,8 \\
(26,3-59,0)\end{array}$ & $\begin{array}{c}89,4 \\
(80,3-94,5)\end{array}$ \\
\hline Fumante & $\begin{array}{c}66.9 \\
(34,5-88,6)\end{array}$ & $\begin{array}{c}95,1 \\
(88,7-98,0)\end{array}$ & $\begin{array}{c}58,5 \\
(29,0-83,0)\end{array}$ & $\begin{array}{c}96,5 \\
(91,0-98,7)\end{array}$ & $\begin{array}{c}49,5 \\
(22,9-76,3)\end{array}$ & $\begin{array}{c}93,2 \\
(85,4-97,0)\end{array}$ & $\begin{array}{c}42,5 \\
(18,7-70,4)\end{array}$ & $\begin{array}{c}94,8 \\
(88,9-97,6)\end{array}$ \\
\hline \multicolumn{9}{|l|}{$\begin{array}{l}\text { Consumo de bebida } \\
\text { alcoólica }\end{array}$} \\
\hline Não consumidor & $\begin{array}{c}49,0 \\
(37,8-60,2)\end{array}$ & $\begin{array}{c}92,1 \\
(88,7-94,6)\end{array}$ & $\begin{array}{c}55,3 \\
(37,1-72,2)\end{array}$ & $\begin{array}{c}84,7 \\
(77,0-90,2)\end{array}$ & $\begin{array}{c}54,1 \\
(42,1-65,7)\end{array}$ & $\begin{array}{c}91,8 \\
(88,4-94,3)\end{array}$ & $\begin{array}{c}53,5 \\
(42,2-64,4)\end{array}$ & $\begin{array}{c}92,0 \\
(89,1-94,2)\end{array}$ \\
\hline Consumidor & $\begin{array}{c}55,3 \\
(37,1-72,2)\end{array}$ & $\begin{array}{c}84,7 \\
(77,0-90,2)\end{array}$ & $\begin{array}{c}42,6 \\
(27,7-58,9)\end{array}$ & $\begin{array}{c}90,3 \\
(84,9-93,9)\end{array}$ & $\begin{array}{c}52,1 \\
(33,7-69,9)\end{array}$ & $\begin{array}{c}84,4 \\
(75,9-90,3)\end{array}$ & $\begin{array}{c}41,2 \\
(25,7-58,4)\end{array}$ & $\begin{array}{c}89,4 \\
(82,8-93,6)\end{array}$ \\
\hline \multicolumn{9}{|l|}{$\begin{array}{l}\text { Estado nutricional } \\
\text { antropométrico }\end{array}$} \\
\hline $\begin{array}{l}\text { Sem excesso de } \\
\text { peso }\end{array}$ & $\begin{array}{c}49,5 \\
(36,7-62,4)\end{array}$ & $\begin{array}{c}92,1 \\
(88,8-94,5)\end{array}$ & $\begin{array}{c}55,4 \\
(42,8-67,4)\end{array}$ & $\begin{array}{c}90,2 \\
(87,2-92,6)\end{array}$ & $\begin{array}{c}57,2 \\
(43,1-70,2)\end{array}$ & $\begin{array}{c}91,3 \\
(87,9-93,8)\end{array}$ & $\begin{array}{c}48,1 \\
(36,1-60,3)\end{array}$ & $\begin{array}{c}93,8 \\
(90,8-95,8)\end{array}$ \\
\hline Sobrepeso & $\begin{array}{c}39,7 \\
(21,3-61,4)\end{array}$ & $\begin{array}{c}86,7 \\
(80,0-91,5)\end{array}$ & $\begin{array}{c}29,6 \\
(15,9-48,3)\end{array}$ & $\begin{array}{c}91,1 \\
(85,6-94,6)\end{array}$ & $\begin{array}{c}36,9 \\
(18,7-59,7)\end{array}$ & $\begin{array}{c}86,4 \\
(79,7-91,2)\end{array}$ & $\begin{array}{c}27,2 \\
(14,1-46,0)\end{array}$ & $\begin{array}{c}90,9 \\
(85,0-94,6)\end{array}$ \\
\hline Obesidade & $\begin{array}{c}59,7 \\
(42,8-74,6)\end{array}$ & $\begin{array}{c}90,1 \\
(82,0-94,8)\end{array}$ & $\begin{array}{c}66,8 \\
(50,3-80,1)\end{array}$ & $\begin{array}{c}87,0 \\
(78,9-92,3)\end{array}$ & $\begin{array}{c}58,5 \\
(42,3-73,0)\end{array}$ & $\begin{array}{c}9,4 \\
(84,1-95,6)\end{array}$ & $\begin{array}{c}72,4 \\
(55,3-84,8)\end{array}$ & $\begin{array}{c}85,1 \\
(77,2-90,6)\end{array}$ \\
\hline \multicolumn{9}{|l|}{$\begin{array}{l}\text { Prática de atividade } \\
\text { física }\end{array}$} \\
\hline $\begin{array}{l}\text { Não cumpre a } \\
\text { recomendação }\end{array}$ & $\begin{array}{c}48,9 \\
(38,2-59,6)\end{array}$ & $\begin{array}{c}89,8 \\
(86,2-92,5)\end{array}$ & $\begin{array}{c}50,4 \\
(39,9-60,8)\end{array}$ & $\begin{array}{c}89,2 \\
(86,0-91,7)\end{array}$ & $\begin{array}{c}55,1 \\
(42,6-67,0)\end{array}$ & $\begin{array}{c}90,1 \\
(86,6-92,7)\end{array}$ & $\begin{array}{c}50,5 \\
(39,3-61,6)\end{array}$ & $\begin{array}{c}91,6 \\
(88,5-94,0)\end{array}$ \\
\hline $\begin{array}{l}\text { Cumpre a } \\
\text { recomendação }\end{array}$ & $\begin{array}{c}53,8 \\
(37,7-69,2)\end{array}$ & $\begin{array}{c}90,9 \\
(85,3-94,5)\end{array}$ & $\begin{array}{c}53,8 \\
(38,5-68,4)\end{array}$ & $\begin{array}{c}90,9 \\
(86,4-94,0)\end{array}$ & $\begin{array}{c}50,7 \\
(34,5-66,8)\end{array}$ & $\begin{array}{c}89,7 \\
(83,7-93,6)\end{array}$ & $\begin{array}{c}47,0 \\
(33,1-61,4)\end{array}$ & $\begin{array}{c}91,0 \\
(85,9-94,4)\end{array}$ \\
\hline \multicolumn{9}{|l|}{ Hipertensão } \\
\hline Sim & $\begin{array}{c}70,13 \\
(59,0-79,3)\end{array}$ & $\begin{array}{c}85,2 \\
(78,8-90,0)\end{array}$ & $\begin{array}{c}66,7 \\
(55,7-76,1)\end{array}$ & $\begin{array}{c}87,1 \\
(81,8-91,1)\end{array}$ & $\begin{array}{c}68,7 \\
(57,4-78,2)\end{array}$ & $\begin{array}{c}84,0 \\
(77,3-89,0)\end{array}$ & $\begin{array}{c}63,3 \\
(52,5-72,9)\end{array}$ & $\begin{array}{c}87,0 \\
(81,7-91,0)\end{array}$ \\
\hline Não & $\begin{array}{c}25,9 \\
(15,5-40,1)\end{array}$ & $\begin{array}{c}92,0 \\
(88,1-94,6)\end{array}$ & $\begin{array}{c}28,6 \\
(16,9-44,0)\end{array}$ & $\begin{array}{c}90,9 \\
(88,1-93,1)\end{array}$ & $\begin{array}{c}30,6 \\
(17,8-47,4)\end{array}$ & $\begin{array}{c}92,0 \\
(88,2-94,7)\end{array}$ & $\begin{array}{c}27,7 \\
(15,8-43,9)\end{array}$ & $\begin{array}{c}93,0 \\
(89,7-95,3)\end{array}$ \\
\hline
\end{tabular}

(continua) 
Tabela 3 (continuação)

\begin{tabular}{|c|c|c|c|c|c|c|c|c|}
\hline \multirow[t]{2}{*}{ Características } & \multicolumn{4}{|c|}{ Colesterol total } & \multicolumn{4}{|c|}{ LDL-C } \\
\hline & $\begin{array}{l}\text { Sensibilidade } \\
\text { (IC95\%) }\end{array}$ & $\begin{array}{c}\text { Especificidade } \\
\text { (IC95\%) }\end{array}$ & $\begin{array}{c}\text { VPP } \\
\text { (IC95\%) }\end{array}$ & $\begin{array}{c}\text { VPN } \\
\text { (IC95\%) }\end{array}$ & $\begin{array}{c}\text { Sensibilidade } \\
\text { (IC95\%) }\end{array}$ & $\begin{array}{l}\text { Especificidade } \\
\text { (IC95\%) }\end{array}$ & $\begin{array}{c}\text { VPP } \\
\text { (IC95\%) }\end{array}$ & $\begin{array}{c}\text { VPN } \\
\text { (IC95\%) }\end{array}$ \\
\hline \multicolumn{9}{|l|}{ Diabetes } \\
\hline Sim & $\begin{array}{c}70,4 \\
(54,0-82,8)\end{array}$ & $\begin{array}{c}72,2 \\
(59,9-81,8)\end{array}$ & $\begin{array}{c}56,5 \\
(40,4-71,3)\end{array}$ & $\begin{array}{c}82,6 \\
(71,4-90,0)\end{array}$ & $\begin{array}{c}61,4 \\
(46,2-74,6)\end{array}$ & $\begin{array}{c}70,0 \\
(56,9-80,5)\end{array}$ & $\begin{array}{c}56,2 \\
(40,0-71,1)\end{array}$ & $\begin{array}{c}74,3 \\
(63,4-82,9)\end{array}$ \\
\hline Não & $\begin{array}{c}43,8 \\
(32,6-55,6)\end{array}$ & $\begin{array}{c}92,5 \\
(89,8-94,6)\end{array}$ & $\begin{array}{c}50,2 \\
(38,2-62,2)\end{array}$ & $\begin{array}{c}90,5 \\
(87,9-92,7)\end{array}$ & $\begin{array}{c}49,8 \\
(37,1-62,5)\end{array}$ & $\begin{array}{c}92,3 \\
(89,5-94,3)\end{array}$ & $\begin{array}{c}46,7 \\
(35,3-58,5)\end{array}$ & $\begin{array}{c}93,1 \\
(90,3-95,1)\end{array}$ \\
\hline \multicolumn{9}{|l|}{$\begin{array}{l}\text { Beneficiário de } \\
\text { plano de saúde }\end{array}$} \\
\hline Sim & $\begin{array}{c}50,8 \\
(40,2-61,3)\end{array}$ & $\begin{array}{c}90,8 \\
(87,5-93,2)\end{array}$ & $\begin{array}{c}57,9 \\
(46,1-69,0)\end{array}$ & $\begin{array}{c}88,0 \\
(84,2-91,0)\end{array}$ & $\begin{array}{c}63,5 \\
(49,7-75,4)\end{array}$ & $\begin{array}{c}88,3 \\
(84,5-91,3)\end{array}$ & $\begin{array}{c}50,9 \\
(40,4-61,3)\end{array}$ & $\begin{array}{c}92,7 \\
(88,7-95,3)\end{array}$ \\
\hline Não & $\begin{array}{c}38,1 \\
(24,9-53,4)\end{array}$ & $\begin{array}{c}93,5 \\
(88,9-96,3)\end{array}$ & $\begin{array}{c}53,9 \\
(35,5-71,3)\end{array}$ & $\begin{array}{c}88,4 \\
(84,1-91,7)\end{array}$ & $\begin{array}{c}35,7 \\
(22,3-51,8)\end{array}$ & $\begin{array}{c}92,5 \\
(87,8-95,5)\end{array}$ & $\begin{array}{c}44,5 \\
(27,7-62,8)\end{array}$ & $\begin{array}{c}89,5 \\
(85,6-92,4)\end{array}$ \\
\hline
\end{tabular}

IC95\%: intervalo de 95\% de confiança; LDL-c: lipoproteína de baixa densidade.

Nota: diferenças significativas verificadas por meio da sobreposição do IC95\% foram destacadas em negrito.

Tabela 4

Fatores associados à sensibilidade da informação de colesterol elevado de adolescentes, adultos e idosos residentes em São Paulo, Brasil, 2015.

\begin{tabular}{|c|c|c|c|c|}
\hline \multirow[t]{2}{*}{ Características } & \multicolumn{2}{|c|}{ Colesterol total } & \multicolumn{2}{|c|}{ LDL-C } \\
\hline & OR & IC95\% & OR & IC95\% \\
\hline Idade (cada 10 anos) & 1,69 & $1,24-2,29$ & 1,71 & $1,13-2,57$ \\
\hline \multicolumn{5}{|l|}{ Tabagismo } \\
\hline Nunca fumou & Referência & - & Referência & - \\
\hline Ex-fumante & 1,98 & $0,68-5,76$ & 3,33 & $1,08-10,27$ \\
\hline Fumante & 3,05 & $0,48-19,56$ & 2,47 & $0,55-11,10$ \\
\hline \multicolumn{5}{|c|}{ Beneficiário de plano de saúde } \\
\hline Não & Referência & - & Referência & - \\
\hline Sim & 2,91 & $1,06-7,99$ & 3,64 & $1,10-12,08$ \\
\hline
\end{tabular}

IC95\%: intervalo de 95\% de confiança; LDL-c: lipoproteína de baixa densidade; OR: odds ratio.

Nota: modelos ajustados por sexo, escolaridade do chefe de família, renda familiar per capita, consumo de álcool, hipertensão e diabetes.

lato de colesterol elevado em população de área rural na Austrália, verificaram sensibilidade, especificidade, VPP e VPN de 44,4\%, 91,2\%, 55,4\% e 87\%, respectivamente. No Brasil, não há estudo de base populacional que avalie a validade dessa informação. Bittencourt et al. 26, ao investigarem a validade de um inquérito de risco referido para doença arterial coronariana em 306 servidores públicos de Juiz de Fora, Minas Gerais, verificaram que a sensibilidade da informação de colesterol total autorreferida foi de 50,3\%, a especificidade de 76,2\%, o VPP de 69,4\% e o VPN de 58,9\%. Dados mais recentes provenientes de estudo que incluiu 14.648 servidores públicos indicaram que $58,8 \%$ dos indivíduos conheciam o diagnóstico de colesterol elevado, utilizando a dosagem de LDL-c como padrão-ouro 9. É possível notar que os indicadores da validade da informação de colesterol elevado autorreferida variam conforme as populações avaliadas, entretanto, de forma geral, observam-se baixa sensibilidade e elevada especificidade dessa informação. Vale destacar que o VPP também foi baixo na população 
estudada quando comparado a outras populações. Tal resultado sugere falta de compreensão sobre o colesterol elevado por parte dos residentes de São Paulo, já que muitas pessoas referiram ter colesterol elevado quando o exame laboratorial e o uso de medicamentos não confirmaram o diagnóstico.

Diversos estudos têm demonstrado relação positiva entre a idade e o conhecimento de diversas condições de saúde, incluindo o colesterol elevado. Estudo multicêntrico que avaliou o conhecimento, tratamento e controle do colesterol elevado em 16.207 hispânicos e latinos de 18 a 74 anos, residentes nos Estados Unidos, encontrou que os indivíduos entre 18 e 44 anos tinham maior probabilidade de não conhecer o diagnóstico quando comparados aos indivíduos com 45 anos ou mais 27. Dados do NHANES de 1988 a 1994 indicaram que a sensibilidade foi significativamente maior entre os indivíduos de 35 anos ou mais quando comparados aos com menos de 35 anos e, após ajuste por variáveis de confusão, a idade foi um dos determinantes da sensibilidade da informação autorreferida de colesterol elevado ${ }^{12}$. No presente estudo, foram encontrados valores baixos de sensibilidade, especialmente para os adolescentes, e a idade permaneceu associada à sensibilidade da informação em ambos os modelos de regressão logística ajustados, considerando as classificações de colesterol total e LDL-c como padrão-ouro.

Apesar de ressaltar as limitações da dosagem exclusiva de colesterol total, o Roteiro do Colesterol da Federação Mundial do Coração (World Heart Federation Cholesterol Roadmap) reconhece que é a dosagem que mais se aproxima da realidade de inúmeros países 28. A atualização da Diretriz Brasileira de Dislipidemia e Prevenção da Aterosclerose recomenda a avaliação do colesterol total nos programas de rastreamento populacional a fim de quantificar o risco cardiovascular e adverte que a análise do perfil lipídico é indicada para indivíduos acima dos 10 anos de idade ou a partir dos dois anos, quando a criança apresentar histórico familiar de colesterol elevado e/ou de doença arterial coronariana prematura, ou se apresentar xantomas, arco corneano, fatores de risco (hipertensão arterial, diabetes mellitus, fumo ou obesidade) ou doença aterosclerótica ${ }^{3}$. Estudo de coorte com 4.320 crianças e adolescentes finlandeses verificou que o colesterol e apolipoproteínas avaliados na infância foram associados à aterosclerose subclínica na idade adulta, estimada pela espessura aumentada da camada íntima-média da artéria carótida, diminuição da elasticidade carotídea e função endotelial braquial comprometida 29. Dessa forma, o rastreamento e detecção precoce do colesterol elevado são imprescindíveis à realização de intervenções para evitar a manutenção e agravamento das doenças cardiometabólicas na vida adulta.

As análises do presente estudo sugerem que ser beneficiário de plano de saúde foi determinante para o conhecimento do diagnóstico de colesterol elevado. O mesmo resultado foi constatado no estudo ELSA-Brasil, com 14.648 servidores públicos de Belo Horizonte, Porto Alegre, Rio de Janeiro, Salvador, São Paulo e Vitória 9 . Estudo realizado em 2003, com amostra representativa de residentes na Região Metropolitana de Belo Horizonte, observou prevalências de usos de serviços preventivos, incluindo a dosagem de colesterol, significativamente maiores entre os indivíduos beneficiários de planos de saúde 30 . Dados da PNS reforçam esses achados, brasileiros com plano de saúde utilizaram mais os serviços de saúde, internação e consulta médica quando comparados aos sem plano de saúde em 2013 31. Dessa forma, é possível que o plano de saúde tenha sido decisivo para o conhecimento do colesterol elevado no presente estudo por causa do maior acesso médico por parte dos beneficiários, permitindo o diagnóstico de colesterol elevado pelo profissional médico, conforme a pergunta utilizada para coleta dessa informação.

A prevalência de beneficiários de planos de saúde no Brasil foi estimada em $41,8 \%$ pelos dados do VIGITEL de 2008 32. Na população de São Paulo, a prevalência de quem é ou já foi beneficiário de plano de saúde chegou a $61,7 \%$ em 2015. Relatório que utilizou informações provenientes do NHANES de 2003 a 2012 verificou que o uso de medicação para reduzir o colesterol em indivíduos de 40 a 64 anos com plano de saúde foi três vezes maior quando comparado aos indivíduos sem plano de saúde, $23,9 \%$ vs. $8,1 \%$, respectivamente 33 . No presente estudo, dos indivíduos que conheciam o diagnóstico, 75,7 e 79,6\% faziam uso de medicação hipolipemiante, considerando o diagnóstico por meio do colesterol total e do LDL-c, respectivamente. Investigação mediante dados de pesquisas nacionais de saúde de oito países de renda média e alta observou que a proporção de pessoas que referiu o diagnóstico de colesterol elevado, mas não utilizavam medicação hipolipemiante, variou de $9 \%$ na Tailândia a $53 \%$ no Japão ${ }^{34}$. Portanto, os resultados aqui apresentados sugerem que a cobertura da medicação para tratamento de colesterol elevado é relativamente alta no Município de São Paulo. 
De acordo com a Diretriz Brasileira de Dislipidemias e Prevenção da Aterosclerose, o uso das estatinas é a primeira opção na prevenção primária e secundária das dislipidemias. As estatinas promovem a redução dos níveis de colesterol sanguíneos com potencial de influenciar todo o conjunto das lipoproteínas circulantes ${ }^{3}$. No Brasil, o programa Aqui tem Farmácia Popular realizado pelo Governo Federal em parceria com o setor privado varejista farmacêutico, tem como finalidade oferecer alternativas de acesso a medicamentos, facilitando o tratamento de doenças importantes para a população, entre elas as dislipidemias. São disponibilizados medicamentos que contêm o princípio ativo sinvastatina 35.

A prevalência de colesterol elevado encontrada no presente estudo é semelhante à reportada por outros estudos nacionais. O Estudo de Riscos Cardiovasculares em Adolescentes (ERICA), de base escolar conduzido entre 2013 e 2014, verificou que uma parcela significativa dos adolescentes brasileiros apresenta alterações dos lipídeos plasmáticos: o colesterol total elevado foi encontrado em 20,1\% dos adolescentes e o LDL-c elevado em 3,5\% 36. Dados recentes do VIGITEL 2016 indicam que a frequência de adultos que referiram diagnóstico médico prévio de dislipidemia foi de $22,6 \%$ e o diagnóstico da doença tornou-se mais comum com o avanço da idade e, no sexo feminino, a tendência foi de diminuição com o aumento do nível de escolaridade 8 .

Uma limitação importante de estudos de validação do colesterol elevado autorreferido é o viés de classificação. A variação biológica intraindividual dos lipídios plasmáticos é considerável, especialmente nos indivíduos que apresentam valores próximos aos limites de classificação. O coeficiente de variação, valor que expressa essas diferenças, é cerca de 10\% para as dosagens de colesterol total e LDL-c. Apesar da recomendação de repetição do exame para confirmação de alterações no perfil lipídico ${ }^{3}$, não foi possível coletar uma nova amostra para realizar uma segunda dosagem no presente estudo. É preciso considerar que se trata de um estudo de base populacional, cujo orçamento inviabiliza tais procedimentos. Contudo, a realização das análises com uso de mais de uma variável do perfil lipídico, colesterol total e LDL-c, fortalece a confiabilidade dos resultados.

Erros de classificação também podem ter ocorrido ao se classificar como "com colesterol elevado" indivíduos com níveis de colesterol total ou LDL-c dentro da normalidade, mas em uso de medicamentos para tratamento dessas condições. Medicamentos podem ser utilizados para outras finalidades além da indicação primária. No entanto, não os considerar também pode ser um importante viés de classificação, visto que o indivíduo pode estar com níveis sanguíneos normais por conta do uso da medicação. Ressalta-se que, assim como em outros estudos de validação 12,24,26,27, as classificações de indivíduos "com colesterol elevado" ou "com diabetes" foram baseadas nos tratamentos farmacológicos estabelecidos em diretrizes vigentes no período em que o estudo foi realizado 22,23.

A pergunta utilizada para coletar a informação do colesterol elevado também pode ser considerada uma fonte de erro, pois restringe o conhecimento da condição ao diagnóstico médico. Por outro lado, a restrição do profissional médico pode aumentar a acurácia da estimativa, impossibilitando que indivíduos não profissionais da área de saúde façam o diagnóstico. Ressalta-se que pesquisas nacionais de base populacional, como o VIGITEL e a PNS, também utilizam o diagnóstico médico para coletar informações sobre morbidade referida 7,8 .

Outra importante limitação foi o pequeno número absoluto de indivíduos diagnosticados com colesterol total e LDL-c elevados, o que restringiu as variáveis de ajuste nos modelos de regressão múltiplos e, em alguns casos, produziu grandes intervalos de confiança. A mesma limitação foi encontrada em estudos anteriores de validação da informação de colesterol elevado autorreferida 24,26. Apesar das limitações, é importante ressaltar que os resultados são provenientes de um estudo de base populacional, com amostra representativa de adolescentes, adultos e idosos do Município de São Paulo. Além disso, tendo em vista a importância do colesterol elevado como fator de risco para doenças cardiometabólicas, destaca-se a relevância de conhecer a validade da informação autorreferida do colesterol elevado, utilizada em diversos inquéritos populacionais.

Os resultados do presente estudo indicam baixa sensibilidade e VPP para a informação de colesterol elevado autorreferida em adolescentes, adultos e idosos residentes em São Paulo. Destaca-se que indivíduos mais velhos, ex-fumantes e beneficiários de planos de saúde souberam referir a condição de hipercolesterolemia quando comparados aos mais jovens, não fumantes e não beneficiários de planos de saúde. 


\section{Colaboradores}

M. M. Fontanelli, L. R. Nogueira, M. R. Garcez e C. H. Sales contribuíram na análise e interpretação dos dados e redação do manuscrito. J. E. Corrente contribuiu na análise de dados e revisou criticamente o conteúdo intelectual do manuscrito. C. L. G. César e M. Goldbaum contribuíram na concepção e delineamento do estudo e revisaram criticamente o conteúdo intelectual do manuscrito. R. M. Fisberg contribuiu na concepção e delineamento do estudo, análise e interpretação de dados e revisou criticamente o conteúdo intelectual do manuscrito. Todos os autores aprovaram a versão final apresentada e são responsáveis pela garantia da exatidão e integridade de qualquer parte da obra.

\section{Agradecimentos}

Agradecemos à equipe do ISA-Capital 2015, especialmente à Maria Cecília Goi Porto Alves, aos pesquisadores do Grupo de Avaliação do Consumo Alimentar e a todos os voluntários que abriram as portas de suas casas para que se realizasse esta pesquisa. Secretaria Municipal de Saúde de São Paulo (2013-0.235.936-0), Fundação de Amparo à Pesquisa de São Paulo (2012/22113-9), Conselho Nacional de Desenvolvimento Científico e Tecnológico (472873/2012-1).

\section{Referências}

1. GBD 2015 DALYs and HALE Collaborators. Global, regional, and national disability-adjusted life-years (DALYs) for 315 diseases and injuries and healthy life expectancy (HALE), 1990-2015: a systematic analysis for the Global Burden of Disease Study 2015. Lancet 2016; 388:1603-58.

2. Cooney MT, Dudina AL, Graham IM. Value and limitations of existing scores for the assessment of cardiovascular risk: a review for clinicians. J Am Coll Cardiol 2009; 54:1209-27.

3. Faludi AA, Izar MCO, Saraiva JFK, Chacra APM, Bianco HT, Afiune Neto A, et al. Atualização da Diretriz Brasileira de Dislipidemias e Prevenção da Aterosclerose - 2017. Arq Bras Cardiol 2017; 109(2 Suppl 1):1-76.

4. Prospective Studies Collaboration. Blood cholesterol and vascular mortality by age, sex, and blood pressure: a meta-analysis of individual data from 61 prospective studies with 55,000 vascular deaths. Lancet 2007; 370:1829-39.

5. Rose G. Strategy of prevention: lessons from cardiovascular disease. Br Med J (Clin Res Ed) 1981; 282:1847-51.

6. World Health Organization. Global Health Observatory (GHO) data - raised cholesterol. Geneva: World Health Organization; 2017.

7. Lotufo PA, Santos RD, Sposito AC, Bertolami M, Rocha-Faria Neto J, Izar MC, et al. Selfreported high-cholesterol prevalence in the Brazilian population: analysis of the $2013 \mathrm{Na}$ tional Health Survey. Arq Bras Cardiol 2017; 108:411-6.

8. Departamento de Vigilância de Doenças e Agravos não Transmissíveis e Promoção da Saúde, Secretaria de Vigilância em Saúde, Ministério da Saúde. Vigitel Brasil 2016: vigilância de fatores de risco e proteção para doenças crônicas por inquérito telefônico: estimativas sobre frequência e distribuição sociodemográfica de fatores de risco e proteção para doenças crônicas nas capitais dos 26 estados brasileiros e no Distrito Federal em 2016. Brasília: Ministério da Saúde; 2017.

9. Lotufo PA, Santos RD, Figueiredo RM, Pereira AC, Mill JG, Alvim SM, et al. Prevalence, awareness, treatment, and control of high low-density lipoprotein cholesterol in Brazil: baseline of the Brazilian Longitudinal Study of Adult Health (ELSA-Brasil). J Clin Lipidol 2016; 10:568-76.

10. Lanas F, Serón P, Lanas A. Coronary heart disease and risk factors in Latin America. Glob Heart 2013; 8:341-8.

11. Silva H, Hernandez-Hernandez R, Vinueza R, Velasco M, Boissonnet CP, Escobedo J, et al. Cardiovascular risk awareness, treatment, and control in urban Latin America. Am J Ther 2010; 17:159-66.

12. Natarajan S, Lipsitz SR, Nietert P. Self-report of high cholesterol: determinants of validity in U.S. adults. Am J Prev Med 2002; 23:13-21. 
13. Fisberg RM, Sales CH, Fontanelli MM, Pereira JL, Alves MCGP, Escuder MML, et al. 2015 Health Survey of São Paulo with focus in nutrition: rationale, design, and procedures. $\mathrm{Nu}-$ trients 2018; 10:E169.

14. Alves MCGP, Escuder MML, Goldbaum M, Barros MBA, Fisberg RM, Cesar CLG. Smapling plan in health surveys, city of São Paulo, Brazil, 2015. Rev Saúde Pública 2018; 52:81.

15. Matsudo S, Araújo T, Matsudo V, Andrade D, Andrade E, Oliveira LC, et al. Questionário Internacional de Atividade Física (IPAQ): estudo de validade e reprodutibilidade no Brasil. Rev Bras Ativ Fís Saúde 2001; 2:5-18.

16. World Health Organization. Global recommendations on physical activity for health. Geneva: World Health Organization; 2010.

17. World Health Organization. Physical status: the use and interpretation of anthropometry. Report of a WHO Expert Committee. Geneva: World Health Organization; 1995.

18. World Health Organization. WHO Child and Growth Standards: length/height-for-age, weight-for-age, weight-for-length, weight-forheight and body mass index-for-age: methods and development. Geneva: World Health Organization; 2006.

19. World Health Organization. WHO Consultation on Obesity: preventing and managing the global epidemic. Geneva: World Health Organization; 1998. (WHO Technical Report Series, 894).

20. LebrãoML, DuarteYAO, organizadoras. SABE Saúde, Bem-estar e Envelhecimento. O projeto Sabe no município de São Paulo: uma abordagem inicial. Brasília: Organização Pan-Americana da Saúde; 2003.

21. Sociedade Brasileira de Cardiologia; Sociedade Brasileira de Hipertensão; Sociedade Brasileira de Nefrologia. VI Diretrizes Brasileiras de Hipertensão. Arq Bras Cardiol 2010; 95(1 Suppl 1): $1-51$.

22. Sociedade Brasileira de Diabetes. Diretrizes da Sociedade Brasileira de Diabetes 2015-2016. São Paulo: A.C. Farmacêutica; 2016.

23. Xavier HT, Izar MC, Faria Neto JR, Assad MH, Rocha VZ, Sposito AC, et al. V diretriz brasileira de dislipidemias e prevenção da aterosclerose. Arq Bras Cardiol 2013; 101(4 Suppl 1):1-22.

24. Tolonen H, Koponen P, Mindell JS, Männistö S, Giampaoli S, Dias CM, et al. Under-estimation of obesity, hypertension and high cholesterol by self-reported data: comparison of selfreported information and objective measures from health examination surveys. Eur J Public Health 2014; 24:941-8.

25. Newell S, Girgis A, Sanson-Fisher R, Ireland M. Accuracy of patients' recall of Pap and cholesterol screening. Am J Public Health 2000; 90:1431-5.
26. Bittencourt RJ, Chaves SR, Amado RC, Mendonça VF, Oliveira FJF, Antunes CMF. Validação de inquérito de risco referido para vigilância em saúde de fatores de risco de doença arterial coronariana em servidores públicos estaduais de Juiz de Fora, Minas Gerais, Brasil. Cad Saúde Pública 2004; 20:761-70.

27. Rodriguez CJ, Cai J, Swett K, González HM, Talavera GA, Wruck LM, et al. High cholesterol awareness, treatment, and control among Hispanic/Latinos: results from the Hispanic Community Health Study/Study of Latinos. J Am Heart Assoc 2015; 4:e001867.

28. Murphy A, Faria-Neto JR, Al-Rasadi K, Blom D, Catapano A, Cuevas A, et al. World Heart Federation cholesterol roadmap. Glob Heart 2017; 12:179-97.e5.

29. Juonala M, Viikari JS, Raitakari OT. Main findings from the prospective Cardiovascular Risk in Young Finns Study. Curr Opin Lipidol 2013; 24:57-64.

30. Lima-Costa MF. Estilos de vida e uso de serviços preventivos de saúde entre adultos filiados ou não a plano privado de saúde (Inquérito de Saúde de Belo Horizonte). Ciênc Saúde Coletiva 2004; 9:857-64.

31. Malta DC, Bernal RTI, Lima MG, Araújo SSC, Silva MMA, Freitas MIF, et al. Doenças crônicas não transmissíveis e a utilização de serviços de saúde: análise da Pesquisa Nacional de Saúde no Brasil. Rev Saúde Pública 2017; 51 Suppl 1:4s

32. Malta DC, Moura EC, Oliveira M, dos Santos FP. Health insurance users: self-reported morbidity and access to preventive tests according to a telephone survey, Brazil, 2008. Cad Saúde Pública 2011; 27:57-66.

33. Gu Q, Paulose-Ram R, Burt VL, Kit BK. Prescription cholesterol-lowering medication use in adults aged 40 and over: United States, 2003-2012. NCHS Data Brief 2014; (177):1-8.

34. Roth GA, Fihn SD, Mokdad AH, Aekplakorn W, Hasegawa T, Lim SS. High total serum cholesterol, medication coverage and therapeutic control: an analysis of national health examination survey data from eight countries. Bull World Health Organ 2011; 89:92-101.

35. Ministério da Saúde. Portaria no 111, de 28 de janeiro de 2016. Dispõe sobre o Programa Farmácia Popular do Brasil (PFPB). Diário Oficial União 2016; 29 jan.

36. Faria Neto JR, Bento VF, Baena CP, Olandoski M, Gonçalves LG, Abreu GA, et al. ERICA: prevalence of dyslipidemia in Brazilian adolescents. Rev Saúde Pública 2016; 50 Suppl 1:10s. 


\section{Abstract}

The study aimed to validate self-report of high cholesterol in São Paulo, Brazil, and verify factors associated with this information's sensitivity. Data were used from the Health Survey of the City of São Paulo 2015, a cross-sectional population-based study with a probabilistic sample of the city's residents. The sample included 886 individuals with information from the structured questionnaire, blood measurements of total cholesterol and fractions or who reported being on medication for high cholesterol. The validity of self-reported information on high cholesterol was measured according to sensitivity, specificity, positive predictive value (PPV), negative predictive value (NPV), and kappa index, considering total cholesterol and LDL-c and pharmacological treatment as the gold standard. Logistic regression models were developed to investigate factors associated with the sensitivity of this information. The sensitivity of information on high cholesterol using total cholesterol as the reference was $50.6 \%$, specificity 90.19\%, PPV 51.64\%, NPV 89.82\%, and kappa 0.41. Taking $L D L-c$ as the gold standard, sensitivity was $53.52 \%$, specificity $89.93 \%, P P V \quad 49.22 \%$, NPV 91.39\%, and kappa 0.43. Using total cholesterol as the reference, age $(\mathrm{OR}=1.69 ; 95 \% \mathrm{CI}$ : 1.24-2.29) and having a private health plan $(\mathrm{OR}=2.91 ; 95 \% \mathrm{CI}: 1.06-7.99)$ were associated with the information's sensitivity. With $L D L-c$ as the gold standard, age $(O R=1.71 ; 95 \% \mathrm{CI}: 1.13-$ 2.57), smoking (OR $=3.33$; 95\%CI: 1.08-10.27), and having a private health plan $(O R=3.64$; 95\%CI: 1.10-12.08) were associated with the information's sensitivity. The results suggest low sensitivity and low PPV of self-reported high cholesterol in residents of São Paulo.

Cholesterol; LDL Cholesterol;

Hypercholesterolemia; Validation Studies

\section{Resumen}

El objetivo de este estudio fue validar el autoinforme de colesterol elevado en residentes de São Paulo, Brasil, y verificar los factores asociados a la sensibilidad de esta información. Se utilizaron datos de la Encuesta de Salud de São Paulo 2015, estudio transversal de base poblacional con una muestra probabilística de residentes del municipio. Se incluyeron 886 individuos con información del cuestionario estructurado, cantidad de colesterol total y fracciones o que informaron de un tratamiento farmacológico por colesterol elevado. La validez de la información autoinformada de colesterol elevado fue determinada por medio de la sensibilidad, especificidad, valores predictivos positivo (VPP) y negativo (VPN) y kappa, considerando las cantidades de colesterol total y LDL-c y el tratamiento farmacológico como parámetro de referencia. Se realizaron modelos de regresión logística, a fin de investigar los factores asociados a la sensibilidad de esa información. La sensibilidad de la información de colesterol elevado, teniendo en cuenta el colesterol total como referencia, fue de un 50,6\%, la especificidad de 90,19\%, VPP de 51,64\%, VPN de 89,82\% y kappa de 0,41. Considerando el $L D L-c$ como parámetro de referencia, la sensibilidad fue 53,52\%, la especificidad 89,93\%, el VPP 49,22\%, el VPN 91,39\% y kappa 0,43. Utilizando la cantidad de colesterol total como referencia, la edad $(O R=1,69$; IC95\%: 1,24-2,29) y el plan de salud $(O R=2,91$; IC95\%: 1,06-7,99) se asociaron a la sensibilidad de esa información. Considerando la cantidad de $L D L-c$ como parámetro de referencia, la edad (OR = 1,71; IC95\%: 1,13-2,57), el tabaquismo $(O R=3,33$; IC95\%: 1,08-10,27) y el plan de salud (OR = 3,64; IC95\%: 1,10-12,08) se asociaron a la sensibilidad de esa información. Los resultados indican baja sensibilidad y VPP del autoinforme de colesterol elevado en residentes de São Paulo.

Colesterol; LDL-Colesterol; Hipercolesterolemia; Estudios de Validación
Recebido em 26/Fev/2018

Versão final reapresentada em 01/Ago/2018

Aprovado em 17/Ago/2018 